九州大学学術情報リポジトリ

Kyushu University Institutional Repository

\title{
Stimulation by Natural Rubber Serum Powder of the Growth of Bifido bac terium bifidum
}

Oiki, Hirokazu

Laboratory of Microbial Technology, Department of Food Science and Technology, Faculty of Agriculture, Kyushu University

Sonomoto, Kenj i

Laboratory of Microbial Technology, Department of Food Science and Technology, Faculty of Agriculture, Kyushu University

Ishizaki, Ayaaki

Laboratory of Microbial Technology, Department of Food Science and Technology, Faculty of Agriculture, Kyushu University

https://doi.org/10.5109/24113

出版情報 : 九州大学大学院農学研究院紀要. 40 (3/4)，pp.271-277，1996-03. Kyushu University バージョン：

権利関係: 


\title{
Stimulation by Natural Rubber Serum Powder of the Growth of Bifido bac terium bifidum
}

\author{
Hirokazu Oiki, Kenji Sonomoto and Ayaaki Ishizaki
}

\author{
Laboratory of Microbial Technology, Department of Food Science and Technology, \\ Faculty of Agriculture, Kyushu University, Fukuoka 812-81, Japan \\ (Received October 17, 1995)
}

\begin{abstract}
Natural rubber serum powder (NRSP) was found to have a remarkable growthstimulating effect on Bifidobacterium bifidum. We compared NRSP with conventional stimulators of the growth of this microorganism, and we found that NRSP had a similar growth-stimulating effect to that of casein, as well as a synergistic effect. However, the effects of the concentration of casein and of NRSP on the growth of $B$. bifidum were different. Ammonium sulfate, which is one of the major contaminants of NRSP, enhanced the growth of the test strain, acting as a nitrogen source, and the other active ingredients of NRSP had additive nutritional effects.
\end{abstract}

\section{INTRODUCTION}

Bifidobacteria are nonmotile, Gram-positive bacteria that are major components of the intestinal flora of healthy humans. These bacteria are considered beneficial for all age groups because they play a significant role in controlling the $\mathrm{pH}$ of the large intestine through the secretion of lactic and acetic acids which, in turn, inhibit the growth of many harmful bacteria such as clostridia. In healthy breast-fed infants, bifidobacteria are the most prevalent genus of bacteria. However, their levels decrease gradually and numbers of some harmful bacteria increase as the host grows older.

Bifidobacteria have been used as food supplements and in milk fermentations (Ishibashi, 1990). Earlier researchers demonstrated that the ingested bifidobacteria may (i) stimulate the existing population of bifidobacteria and (ii) reestablish a population of beneficial microflora after serious depletion by infection (Tamime et al., 1995). Kaloud and Stögmann (1969) demonstrated that the regular intake of B. bifidum by healthy infants provided a some protection against enteric infections. B. bifidum and B. longum are widely employed as mixed starter cultures in combination with Lactobacillus acidophilus and/or other yogurt-generating organisms (Modler et al., 1990; Tamime et al., 1995). However, the use of bifidobacteria for such purposes has been limited because they require bifidogenic growth stimulators (BGSs). Several growth-stimulating substances have been reported (Poch and Bezkorovainy, 1988, 1991; Ibrahim and Bezkorovainy, 1994; Kaneko et al., 1994), most of which are costly and/or have some disadvantages. For example, B. bifidum cannot utilize many kinds of oligosaccharide, such as fructooligosaccharide (Hidaka, 1993) and soybean oligosaccharide (Masai, 1988), which are well known as BGSs.

In our laboratory, we have investigated the application of NRSP, the spray-dried product of the serum obtained during the process for separation of natural rubber latex, as a stimulator of the growth of various anaerobic microorganisms (Ishizaki, 1989; Tripetchkul et al., 1992). Recently, Ishizaki (1995) reported that NRSP had a strong 
growth-stimulatory effect on a wide range of bifidobacteria of human origin. For the application of bifidobacteria to the food industry, further investigations into the effects of NRSP on the growth of B.bifidum are necessary.

The purpose of the work described in this paper was to extend our understanding of the effects of the stimulation by NRSP of the growth of B.bifidum and to compare NRSP with a conventional BGS, such as a digest of bovine casein.

\section{MATERIALS AND METHODS}

\section{Natural rubber serum powder (NRSP)}

NRSP was provided by Nakanihon Air Service Co., Ltd. (Nagoya). NRSP used in this study was the spray-dried product of natural rubber serum imported from Malaysia. It was composed of many amino acids, peptides, inorganic salts and so on. The detailed composition of NRSP was described previously (Ishizaki, 1989).

\section{Microorganism and media}

B. bifidum JCM1254, of human origin, was purchased from the Japan Collection of Microorganisms, Wako.

Bifidobacterium medium, recommended by the Japan Collection of Microorganisms as a complete medium for efficient growth of bifidobacteria, was used for the growth of $B$. bifidum. This mediurn contained (per liter of distilled water, $\mathrm{pH}$ 6.8) $10 \mathrm{~g}$ casein (enzymatic hydrolysate; Sigma Co., Ltd., St. Louis, MO, USA), 5 g meat extract, 5 g yeast extract, $10 \mathrm{~g}$ glucose, $1 \mathrm{ml}$ Tween $80,0.5 \mathrm{~g}$ cysteine $\mathrm{HCl}_{2} \mathrm{O}, 3 \mathrm{~g} \mathrm{~K}_{2} \mathrm{HPO}_{+}$and $10 \mathrm{~g}$ ascorbic acid. Glucose, cysteine $\mathrm{HCl} \mathrm{H}_{2} \mathrm{O}$ and ascorbic acid were added aseptically via a disposable filtration unit (DISMIC-25; ADVANTEC Toyo Co., Ltd., Tokyo) before inoculation of cells.

For determinations of the effects of various nutrients on the growth of B.bifidum, we used the following media: medium I, bifidobacterium medium without organic nutrients, namely, casein, meat extract and yeast extract; and medium II, bifidobacterium medium without casein.

\section{Culture and analysis}

The microorganism was grown in anaerobic jars (GasPak; BBL Microbiology Systems, Cockeysville, MD, USA) in thioglycolate (TGC) medium without dextrose (Difco Laboratories, Detroit, MI, USA) and stored at $5^{\circ} \mathrm{C}$.

The stored culture was precultured in the bifidobacterium medium at $37^{\circ} \mathrm{C}$ for $12-15$ $\mathrm{h}$. Forty $\mathrm{ml}$ of the experimental medium were inoculated with $5 \%(\mathrm{v} / \mathrm{v})$ of the preculture. The cultures were incubated in ZOO-ml Erlenmeyer flask, with shaking at 72 strokes/min, at $37^{\circ} \mathrm{C}$ under anaerobic conditions. Air was replaced by a mixture of $\mathrm{N}_{2}, \mathrm{H}_{2}$ and $\mathrm{CO}$, $(75: 20: 5, \mathrm{v} / \mathrm{v})$. The extent of growth was determined by measuring the absorbance of $\mathbf{5 6 2}$ $\mathrm{nm}\left(\mathrm{A}_{\mathrm{Fi}}\right)$ at various time intervals and the dry cell weight $(\mathrm{DCW})$ was calculated by reference to a previously constructed standard curve. In order to standardize the reporting of the results, all growth experiments in medium I and medium II were accompanied by culture of the microorganism in the complete bifidobacterium medium since growth was not necessarily reproducible from one day to another. 


\section{RESULTS}

\section{Effects of NRSP on the growth of B.bifidum}

Figure 1 shows details of the growth of B. bifidum in the bifidobacterium medium and in bifidobacterium medium supplemented with $1 \%(\mathrm{w} / \mathrm{v})$ NRSP. The maximum DCW obtained in NRSP-supplemented bifidobacterium medium was higher than that in the bifidobacterium medium alone. Furthermore, NRSP enhanced the growth rate of the cells, as shown in Figure 1. As shown in Figure 2, the test strain grew poorly in medium I, as expected from the fact that medium I contained no organic nutrients. By contrast, it is clear that NRSP had a remarkable growth-stimulating effect even in minimal medium such as medium $\mathrm{I}$.

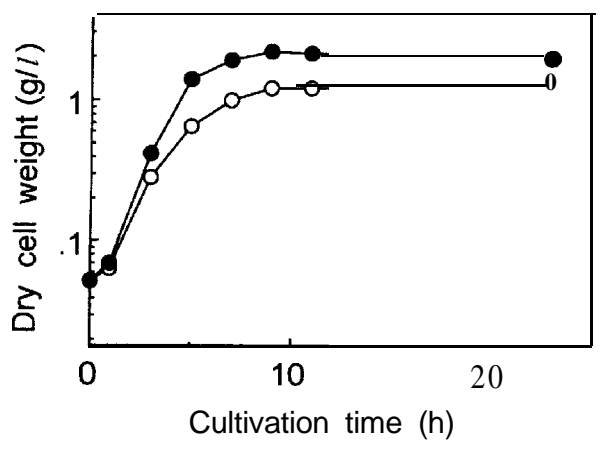

Fig. 1. Effects of NRSP in bifidobacterium medium on the cell growth of Bifidobacterium bifidum JCM 1254. Growth of the microorganism is expressed in terms of dry cell weight per litrr of medium plus $10 \mathrm{~g} / \mathrm{l}$ NRSP (O) or without NRSP (0).

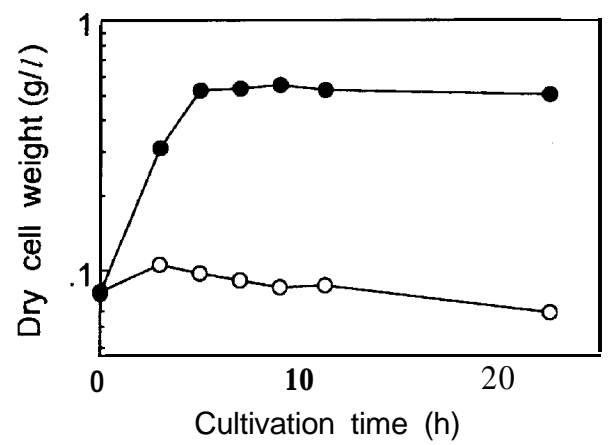

Fig. 2. Effects of NRSP in minimal medium (medium I) on the cell growth of Bifidobacteriumbifidum JCM 1254. Growth of the microorganism is expressed as in Fig. 1. Medium I with $10 \mathrm{~g} / \mathrm{NRSP}(\mathrm{O})$ and without NRSP (O) was used as the experimental medium. 


\section{Effects of NRSP as compared to those of other nutrients}

For a further understanding of the growth-stimulating effect of NRSP, we compared the growth of B. bifidum in medium I supplemented with NRSP and/or the organic nutrients of the bifidobacterium medium, namely, casein, meat extract and yeast, extract. The results are shown in Figure 3. As seen from this Figure, NRSP had a good growthstimulating effect on the cells in all cases in all media tested in this series of experiments. Casein also stimulated the growth of the microorganism, while addition of meat extract and yeast extract to medium I had no significant effect. However, these extracts increased the maximum DCW in the presence of casein and/or NRSP. The growth rate in all cases tested reflected the maximum DCW obtained.

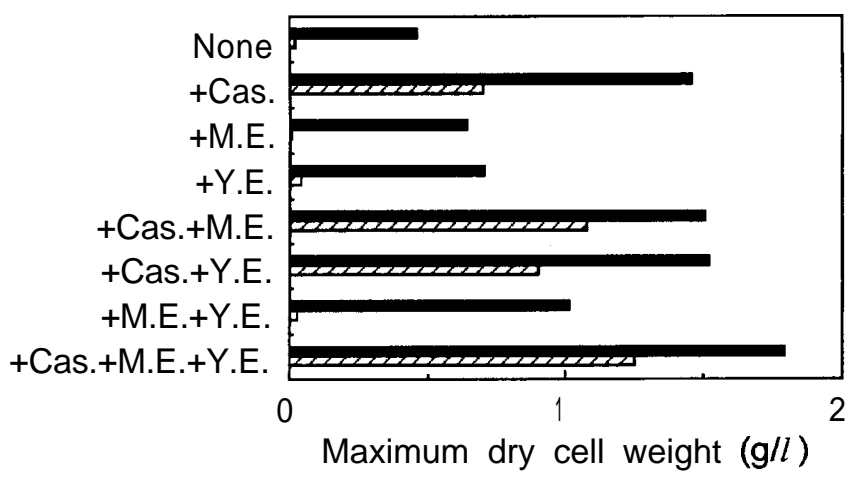

Fig. 3. Effects of NRSP in combination with various organic nutrients. Hatched bars indicate the growth in medium without NRSP and black bars indicate the growth in medium with $1 \%$ NRSP. Medium I was supplemented with various combinations of organic nutrients as follows: +Cas., $1 \%$ casein; +M.E., $0.5 \%$ meat extract; +Y.E., $0.5 \%$ yeast extract.

\section{Effects of various concentrations of NRSP and casein}

NRSP had a growth-stimulatory effect similar to that of casein, which was essential for growth of the test strain as described above. We investigated the effect of the concentration of NRSP and of casein on the growth of B.bifidum (Fig. 4). Different amounts of NRSP were added to medium II, which is described in Materials and Methods. Growth of the test strain was promoted as the concentration of NRSP was increased, with a maximum value at $4 \%$ NRSP. The growth-stimulatory effects of casein also increased with increases in the concentration of casein.

\section{Effects of NRSP and ammonium sulfate}

Ammonium sulfate is a contaminant of NRSP that is introduced during the manufacturing process (Ishizaki, 1989). It is present at a concentration of about $25 \%$ (w/w NRSP). We cannot ignore the possibility of an effect of ammonium sulfate since various strains of Bifidobacterium can be grown in a simplified medium that contains ammonium sulfate as the sole source of nitrogen (Tanaka and Mutai, 1980). Figure 5 


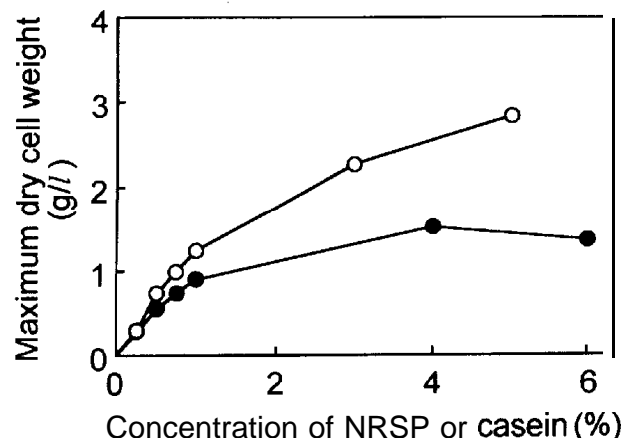

Fig. 4. Effects of concentrations of NRSP and casein on the growth of Bifidobacterium bifidum JCM 1254. Medium II was supplemented with the indicated concentrations of NRSP $(O)$ and casein $(O)$.

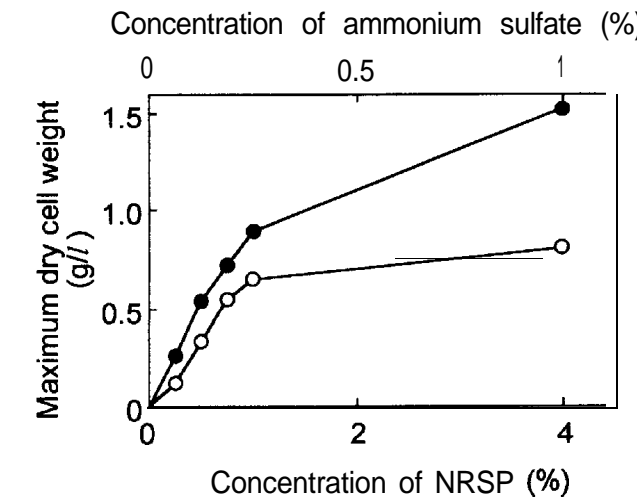

Fig. 5. Effects of various concentrations of NRSP and ammonium sulfate on the growth of Bifidobacterium bifidum JCM 1254. Medium II was supplemented with the indicated concentrations of NRSP (O) and ammonium sulfate $(\bigcirc)$.

shows the effects of various concentrations of NRSP and ammonium sulfate. The maximum DCW was barely increased by addition of ammonium sulfate above $0.25 \%$, which corresponds to a concentration of $1 \%$ of NRSP. However, NRSP had a strong growth-stimulatory effects even at $4 \%$, which is equivalent to $1 \%$ of ammonium sulfate.

\section{DISCUSSION}

The purpose of this study was to investigate the effects of NRSP on the growth of $\mathbf{B}$. bifidum. In a previous paper (Ishizaki, 1995), we indicated that NRSP had strong 
growth-stimulator-y effects on many species of Bifidobacterium. Further investigations have been made using $B$. infantis because this strain has a growth rate that is almost the average of those of all strains tested previously. However, Yuhara et al.(1983) and Alm (1991) noted that B. bifidum is one of the most frequent isolates from breast-fed infants. In addition, Benno and Mitsuoka (1989) demonstrated that $B$. brave is the predominant isolate from the feces of breast-fed infants, These inconsistent data are attributable to the growth of the microorganisms in different environments.

Bifidobacterium bifidum is widely employed in mixed starter cultures in combination with Lactobacillus acidophilus and/or yogurt-generating organisms (Modler et al., 1990; Tamime et al., 1995): for example, Biograde" (Germany), Ofilus (France), Cultura ${ }^{k}$ (Denmark) and Mil-Mil ${ }^{\bar{k}}$ (Japan). For the application of bifidobacteria to the food industry, further investigations of $B$. bifidum are necessary.

Figure 1 shows that NRSP can enhance the growth rate of $B$. bifidum and the cell mass obtained, no matter how rich the growth medium. Furthermore, this compound supported good growth of the test strain in medium I, which did not contain any of the organic nutrients required by Bifidobacterium species (Fig. 2). Thus, NRSP can be utilized as the sole source of organic nutrients, that is, not only as a growth stimulator but also as a source of nitrogen by $B$. bifidum.

It is known that casein is a typical stimulator of the growth of Bifidobacterium species (Kehagias et al., 1977). Casein had a strong growth-stimulating effect on $B$. bifidum in medium I (Fig. 3). Thus, casein was also utilized not only as a BGS but also as a nitrogen source. By contrast, the test strain failed to grow in medium I supplemented with meat extract and yeast extract as the sole organic nutrients (Fig. 3). These extracts did, however, have moderate growth-stimulating effects on $B$. bifidum upon addition to the synthetic medium that contained a nitrogen source, vitamins, nucleic acids and no BGS (data not shown). Thus, both meat and yeast extracts might be utilized as growth stimulators, but not as nitrogen sources by B. bifidum. Poch and Bezkorovainy (1988) suggested that yeast extract has a growth-stimulating effect on various species in the genus Bifidobacterium including $B$. bifidum.

Figure 3 shows that NRSP had a synergistic effect in all combinations with other nutrients. This kind of synergism was also observed for casein and other components of the bifidobacterium medium, that is, meat extract and yeast extract, (hatched bars in Fig. 3). Effects of the concentration of NRSP on the growth of the test strain differed, however, from those of casein (Fig. 4). Thus, NRSP probably contains different types of BGS from those in casein. As seen in Figure 5, effects of concentrations of NRSP and ammonium sulfate on the growth of B. bifidum differed from one another. It is also clear that ammonium sulfate, one of the major constituents of NRSP, albeit a contaminant, was utilized by the test strain and additive nutritional effects of the other active ingredients of NRSP can be expected.

The results presented herein indicate that NRSP has strong growth-stimulating effects on B. bifidum. However, it is perhaps inappropriate to characterize NRSP as a BGS because of the complexity of the experimental media. It is now necessary to confirm the growth-stimulating effects of NRSP in a synthetic medium and to identify the components that act as BGSs in NRSP. 


\section{REFERENCES}

Alm, L. 1991 The therapeutic effects of various cultures - an overview. In "Therapeutic Properties of Fermented Milks" ed. by R. K. Robinson, Elsevier Applied Science Publishers, London, pp. 45-64

Benno, Y. and T. Mitsuoka 1989 Human intestinal flora in health and in disease. In "Ecology and Role of Intestinal Flora" ed. by T. Mitsuoka, Japan Scientific Societies Press, Tokyo, pp. 57-81 (In Japanese)

Hidaka, H. 1993 Functional sugar to improve human health, fructooligosaccharide. Gendai Kagaku, 268 : 64-68 (In Japanese)

Ibrahim, S. A. and A. Bezkorovainy 1994 Growth-promoting factors for Bifidobacteriumlongum. J. Food Sci., 59: 189-191

Ishibashi, N. 1990 Application of bifidobacterium for yogurt production. Biseibutsu, 6: 66-73 (In Japanese)

Ishizaki, A 1989 Utilization of natural rubber waste as medium ingredients for fermentation process. Microb. Utiliz. Renuable Res., 6: 235-241

Ishizaki, A 1995 Natural rubber serum which contains the special growth promoter for Bifidobacterium. Biotech. Biosci. Biochem., 59: 1150-1151

Kaloud, H. and W. Stögmann 1969 Eine bifidum-milchnahrung im klonischen Versuch. Archiv fur Kinderheilkunde, 177: 29-35

Kaneko, T., H. Mori, M. Iwata andS. Meguro 1994 Growth stimulator for bifidobacteria produced by Propiobacterium reudenreichii and several intestinal bacteria.J.Dairy Sci., 77: XX-404

Krhagias, C., Y. C. Jao, E. M. Mikilajcik and P. M. T. Hanen 1977 Growth response of Bifidobacterium bifidum to a hydrolytic* product isolated from bovine casein.J. Food Sci., 42: 146-150

Masai T. 1988 Characteristics and application of soybean oligosaccharide. Syokuhin to Kagaku, 30: 98103 (In Japanese)

Modler, H. W., R. C.McKellar and M. Yaguchi 1990 Bifidobactcria and bifidogenic factors. Can. Inst. Food Sci.Technol. J., $23: 29-41$

Poch, M. and A. Bezkorovainy 1988 Growth-enhancing supplements for various species of thegenus Bifidobacterium. J. Dairy Sci., 71: 3214-3221

Poch, Mand A. Bozkorovainy 1991 Bovine milk $\kappa$-caseintrypsin digest is a growth enhancer for the genus Bifidobacterium.J.Agric. Food Chem., 39: 73-77

Tamime, A. Y., V.M. E. Marshall and R. K. Robinson 1995 Microbiological and technological aspects of milks fermented by bifidobacteria.J. Dairy Res., 62: 151-1 87

Tanaka, R. andM. Mutai 1980 Improved medium for selective isolation and enumeration of Bifidobacterium. Appl. En viron. Microbiol. 40: $866-869$

Tripetchkul,S., M. Tonokawa and A. 1shizaki 1992 Ethanol production by Zymornonasmobilis using natural rubber waste as a nutritional source. J.Ferment.Bioeng., 74: 384-388

Yuhara, T., S. Isojima, F. Tsuchiya and T. Mitsuoka 1983 On the intestinal flora of bottle-fed infant. Bifidobacteria and Microflora, 2: 33-39 